\title{
Geometric Hahn-Banach theorem
}

\author{
Thierry Coquand
}

September 7, 2004

In [MP2] is proved in a constructive way the following result: a point $x$ lies in a compact convex set $K$ in a normed space if and only if it lies within any bound for $K$ (a bound for $K$ being intuitively a parallel pair of hyperplanes between which $K$ lies). This is given as an application of the localic form of the Hahn-Banach theorem, proved in a non constructive way in [MP1]. In [CC] we give a direct constructive proof of Hahn-Banach theorem and using this, we can give a much simpler proof of the characterisation of compact convex sets.

This note is organised as follows. First we recall the direct description the weak* topology on the unit ball of the dual of a normed space given in $[\mathrm{CC}]$. We then use this to characterise the convex closed hull of a totally bounded subset.

\section{Description of the unit ball in the dual space}

Let $E$ be a normed vector space (over the rational numbers). We write $x \in N(q)$ to express that the norm of $x$ is $<q$. We describe a complete Heyting algebra by generators and relations. This complete Heyting algebra should be thought of as a point-free description of of the unit ball of the dual $E^{\prime}$ for the weak* topology. The generators are formal expressions $\lambda x<q$, with $x \in E$, and $q \in \mathbb{Q}$ and the relations are

(1) $[\lambda x<q] \wedge[q<\lambda x]=0$

(2) $[\lambda(x+y)<r+s] \leq[\lambda x<r] \vee[\lambda y<s]$

(3) $1=[\lambda x<1]$ if $x \in N(1)$

where $q<\lambda x$ is defined to be $\lambda(-x)<-q$, together with the continuity axiom

(4) $[\lambda x<q]=\vee_{q^{\prime}<q}\left[\lambda x<q^{\prime}\right]$

Here are simple remarks about $F n(E)$ :

Proposition 1.1 It follows from (1) and (2) that we have

$$
[\lambda x<p] \wedge[\lambda y<q] \leq[\lambda(x+y)<p+q]
$$

If $0<r$ we have $[\lambda(r x)<r p]=[\lambda x<p]$. If $r<s$ we have $1=[\lambda x<s] \vee[r<\lambda x]$. If $x \in N(q)$ we have $1=[\lambda x<q]$. For any $\epsilon>0$ we have

$$
1=\vee_{q}[q<\lambda x] \wedge[\lambda x<q+\epsilon]
$$

Notice that we recover the generators $\lambda x \in(p, q)$ used in [MP1] by defining

$$
\lambda x \in(p, q) \quad={ }_{\text {def }} \quad[p<\lambda x] \wedge[\lambda x<q]
$$


but the use of generators $\lambda x<q$ is the key to get a simple descrition of the frame $F n(E)$ $[\mathrm{CC}]$. In particular, $[\mathrm{CC}]$ provides a direct proof that this frame is compact regular, which is the localic form of Alaoglu's theorem, and of the following result ${ }^{1}$.

Theorem 1.2 In $F n(E)$ we have

$$
1=\left[\lambda y_{0}<q\right] \vee \ldots \vee\left[\lambda y_{m-1}<q\right]
$$

if, and only if, there exists non negative rationals $s_{j}$ such that $\Sigma s_{j}=1$ and $\Sigma s_{j} y_{j} \in N(q)$.

\section{Convex hull}

We suppose given a totally bounded subset $K$ of $E$. We want to characterise the compact convex hull of $K$.

Theorem 2.1 A point $x$ belongs to the closed convex hull of $K$ if, and only if, we have in the theory $F n(E)$ for all $q \in \mathbb{Q}$

$$
[\lambda x<q] \leq \vee_{y \in K}[\lambda y<q]
$$

One possible intuitive reading of this result is that a point belongs to the closed convex hull of $K$ if, and only if, it belongs to all closed regions bounded by hyperplanes that contain $K^{2}$.

Proof. Assume

$$
[\lambda x<q] \leq \vee_{y \in K}[\lambda y<q]
$$

in $F n(E)$. Fix $\epsilon>0$. Since $K$ is totally bounded, there is a finite family $y_{0}, \ldots, y_{m-1}$ of points in $K$ such that for all $y \in K$ we have $y-y_{j} \in N(\epsilon)$ for some $j<m$. This implies

$$
[\lambda x<q] \leq \vee_{j}\left[\lambda y_{j}<q+\epsilon\right]
$$

for all $q \in \mathbb{Q}$. In the theory $F n(E)$ we have for each $j$, by Proposition 1.1

$$
1=\left[\lambda\left(x-y_{j}\right)<2 \epsilon\right] \vee\left[\epsilon<\lambda\left(x-y_{j}\right)\right]
$$

But $(*)$ implies, using Proposition 1.1

$$
\wedge_{j}\left[\epsilon<\lambda\left(x-y_{j}\right)\right]=0
$$

and hence

$$
1=\vee_{j}\left[\lambda\left(x-y_{j}\right)<2 \epsilon\right]
$$

By theorem 1.2 we can conclude that we have non negative $s_{j}$ such that $\Sigma s_{j}=1$ and $\Sigma s_{j}(x-$ $\left.y_{j}\right) \in N(2 \epsilon)$.

It follows that $x$ belongs to the compact convex hull of $K$, as desired.

\footnotetext{
${ }^{1}$ This is obtained by analysing the distributive lattice which is generated by the relations (1),(2),(3). This distributive lattice is normal $[\mathrm{CaC}]$, and the space $F n(E)$ can be defined as the space of maximal points of the spectrum of this distributive lattice.

${ }^{2}$ The statement we give is a little different from the one in [MP2] but seems to correspond in a closer way to the usual statement.
} 


\section{References}

[CC] J. Cederquist and Th. Coquand. Entailment Relations and Distributive Lattices. Proceeding of Logic Colloquium 1998.

[CaC] M. Carral and M. Coste. Normal spectral spaces and their dimensions. J. Pure Appl. Algebra 30 (1983), no. 3, 227-235.

[Joh] P. T. Johnstone. Stone Spaces. Cambridge studies in advanced mathematics 3, 1982.

[MP1] C.J. Mulvey and J.W. Pelletier. A globalization of the Hahn-Banach theorem. Adv. Math. 89 (1991), no. 1, 1-59.

[MP2] C.J. Mulvey and J.W. Pelletier. A geometric characterization concerning compact, convex sets. Math. Proc. Cambridge Philos. Soc. 109 (1991), no. 2, 351-361. 